\title{
Biological equivalence between LDR and PDR in cervical cancer: multifactor analysis using the linear-quadratic model
}

\author{
José Guilherme Couto, MSc', Isabel Bravo, PhD², Rui Pirraco, MSc'12 \\ Instituto Português de Oncologia Francisco Gentil Porto E.P.E. (IPO - Porto), 2Faculdade de Ciências da Universidade do Porto (FCUP), Portugal
}

\begin{abstract}
Purpose: The purpose of this work was the biological comparison between Low Dose Rate (LDR) and Pulsed Dose Rate (PDR) in cervical cancer regarding the discontinuation of the afterloading system used for the LDR treatments at our Institution since December 2009.

Material and methods: In the first phase we studied the influence of the pulse dose and the pulse time in the biological equivalence between LDR and PDR treatments using the Linear Quadratic Model (LQM). In the second phase, the equivalent dose in $2 \mathrm{~Gy} /$ fraction $\left(\mathrm{EQD}_{2}\right)$ for the tumor, rectum and bladder in treatments performed with both techniques was evaluated and statistically compared. All evaluated patients had stage IIB cervical cancer and were treated with External Beam Radiotherapy (EBRT) plus two Brachytherapy (BT) applications. Data were collected from 48 patients (26 patients treated with LDR and 22 patients with PDR).

Results: In the analyses of the influence of PDR parameters in the biological equivalence between LDR and PDR treatments (Phase 1), it was calculated that if the pulse dose in PDR was kept equal to the LDR dose rate, a small therapeutic loss was expected. If the pulse dose was decreased, the therapeutic window became larger, but a correction in the prescribed dose was necessary. In PDR schemes with 1 hour interval between pulses, the pulse time did not influence significantly the equivalent dose. In the comparison between the groups treated with LDR and PDR (Phase 2) we concluded that they were not equivalent, because in the PDR group the total $\mathrm{EQD}_{2}$ for the tumor, rectum and bladder was smaller than in the LDR group; the LQM estimated that a correction in the prescribed dose of $6 \%$ to $10 \%$ was necessary to avoid therapeutic loss.

Conclusions: A correction in the prescribed dose was necessary; this correction should be achieved by calculating the PDR dose equivalent to the desired LDR total dose.

Key words: brachytherapy, cervical cancer, LDR, PDR, EQD 2 , LQM.

\section{Purpose}

Brachytherapy (BT) treatments use radiation sources that are placed inside or surrounding the tumor and, considering the high dose gradient, is possible to administrate high doses in the tumor, protecting the normal tissues surrounding. As access to the tumor is a major factor in BT, the intracavitary approach makes this technique particularly adequate, being used to treat cervical tumors from stage I to IV as unique approach or as a multi-therapeutic scheme with surgery, chemotherapy or EBRT [1, 2]. There has been strong evolution in brachytherapy field in terms of radioactive sources and applicators used, as well as in imaging, such as the acquisition of Magnetic Resonance Images (MRI) for planning that requires the development of MRI compatible applicators [3]. Alternatively, in the planning systems with the introduction of inverse plans that until now were only used in BT for prostate carcinoma [4], showing that this technique is in con- stant investigation and therefore a constant quality control of the procedures is needed in order to assure that the administered treatment is the prescribed one [5].

The purpose of this work was the biological comparison of the Low Dose Rate (LDR) and Pulsed Dose Rate (PDR) BT techniques regarding the discontinuation of the LDR afterloading system used at our Institution since December 2009.

\section{Material and methods}

This study was divided into two parts. In the Phase 1 we analyzed how the equivalent dose varies when some parameters were changed (pulse dose and the pulse time) using the Linear-Quadratic Model (LQM). The objective was to understand how specific parameters may affect the equivalence between these two techniques. Plus, a sensitivity analysis for the $\alpha / \beta$ ratios and the repair half-time $\left(T_{1 / 2}\right)$ was preformed to understand how they affect the response 
of the tissues when the dose rate was changed. For this purpose two LDR schemes to administrate 20 Gy were chosen ( 0.4 and $0.8 \mathrm{~Gy} / \mathrm{h}$ ) and the physical dose that would be equivalent with a PDR treatment was calculated. Then, the relation between the equivalent dose with PDR if different pulse doses were used was plotted and the same procedure was repeated for the pulse time. Phase 2 intended to investigate if the patients treated at our Institution with LDR and PDR had an equivalent biological dose for the complete treatment (EBRT and BT), since the treatments were changed from $0.9 \mathrm{~Gy} / \mathrm{h}$ LDR scheme to $0.7 \mathrm{~Gy} /$ pulse PDR treatment. The objective was to predict whether with the new PDR scheme there would be any differences in terms of tumor control or secondary effects. All patients had stage IIB cervix carcinoma and were submitted to EBRT plus two BT applications. The patients were assigned to groups depending of the BT technique (LDR versus PDR). The total equivalent dose in $2 \mathrm{~Gy}$ / fraction (total $\mathrm{EQD}_{2}$ ) was calculated for the Point $\mathrm{A}$ (prescribed dose), for the rectum and bladder ICRU points, and it was determined if significant differences occurred between the groups.

\section{External radiotherapy technique}

The technique used in the EBRT was 2 Gy/fraction scheme with fixed 3D conformal fields. The dose administrated at point B was always 50 Gy with EBRT. During EBRT, the patients were evaluated by the physician and when the cervix had a diameter inferior to $4 \mathrm{~cm}$ the first BT fraction was applied, then, when the prescribed dose at point A was achieved, EBRT treatment was continued using fields with a median protection. The maximum dose at the Organs at Risk (OAR) was accessed through the planning system Varian ${ }^{\circledR}$ Eclipse ${ }^{\circledR} 8.9$ used at our Institution.

\section{Brachytherapy technique}

The Low Dose Rate (LDR) technique that was analyzed used a train of ${ }^{137} \mathrm{Cs}$ sources. The sources were placed in the treatment site during the entire treatment time. In the Pulsed Dose Rate (PDR) technique an ${ }^{192}$ Ir stepping source was applied to expose the treatment area during a few minutes and stopping the irradiation when the pulse dose was achieved, allowing access to the patients during non-irradiation periods [2].

Standard applicators (Fletcher-type) were applied and the prescription to specific points of the Manchester system (Point A) was made in gray. The dose at the ICRU points for bladder and rectum was calculated. The bladder and rectum points were located using a balloon filled with $7 \mathrm{cc}$ of contrast for the bladder and an opaque marker for the rectum [6]. The dose limit used at our Institution to these Organs at Risk (OAR) was $75 \%$ of the prescribed dose. The dose prescribed in the first BT was determined by the physician after evaluation of the patient reaction to EBRT and through the dose at the Organs at Risk (OAR). We used the Nucletron ${ }^{\circledR}$ Plato $^{\mathrm{TM}}$ version 3.3.5 planning system either for the LDR or PDR treatments.

\section{Dose, time and fractionation}

Although a wide range of documented schemes exists and an extensive variety of fractionation regimens are used in prac- tice, these were based on published schemes, but adapted on empirical or mathematical grounds [2]. The process of the treatment planning for PDR treatments was the same as for LDR. The differences were that, with PDR the stepping source of ${ }^{192}$ Ir was applied, so the dose distribution was not the same as the LDR, because this procedure allows the optimization of the dose distribution by changing the dwell times [7]. According to the ICRU classification, the LDR uses a range of dose rates between 0.4 and $2 \mathrm{~Gy} / \mathrm{h}$, although most of institutions employ a much narrower range $[8,9]$.

In classical Manchester model with ${ }^{226} \mathrm{Ra}$, the dose rate is always $0.5 \mathrm{~Gy} / \mathrm{h}[6,10]$. With the introduction of ${ }^{137} \mathrm{Cs}$, some institutions started to use dose rates between 1.4 and $1.8 \mathrm{~Gy} / \mathrm{h}$. This change in dose rate was accompanied by a decrease in total dose [10]. Mayles calculated that 10 to $15 \%$ of reduction in total dose is mandatory [2]. The American Brachytherapy Society (ABS) recommends a dose rate of 0.5 to $0.65 \mathrm{~Gy} / \mathrm{h}$ to A points, but it is referred that each institution can adjust it in order to respond to specific requirements of the department [11]. Several authors refer that if the pulse dose in PDR is equal or lower than the LDR dose rate, a great therapeutic loss is not expected [12-16]. In the PDR scheme used, a fixed dose rate of $0.7 \mathrm{~Gy} /$ pulse is applied, as the source became older, the pulse time increases due to the lowering of the activity. The influence of the pulse dose and the pulse time in the equivalent dose were analyzed in this study. At our Institution, the intervals between PDR pulses always last for one hour, this interval was assumed in all the calculations performed in this work.

\section{The linear-quadratic model}

The estimation of the effects caused by radiation are very complex - they depend on several factors like total dose, dose rate, fractionation, oxygenation of the tissues, cell sensibility to radiation, etc. which influence the mechanisms of response to the radiation like repair, reassortment, reoxygenation and repopulation [7]. For the assessment of effects caused by radiation, the Linear-Quadratic Model (LQM) is worldwide used. This instrument has a very important role in the decision process when a change in a fractionation scheme is necessary [7]. The $\alpha / \beta$ ratios used in this work were $10 \mathrm{~Gy}$ for tumor and $3 \mathrm{~Gy}$ for late effect tissues. These values are worldwide accepted and are recommended by the GEC ESTRO. Although $\mathrm{T}_{1 / 2}$ is not as consolidated as the previous biological parameters, GEC ESTRO referrers that $1.5 \mathrm{~h}$ is the "best estimate" for this parameter, therefore this is the value that was used; this parameter is less adequate for in situ tumors which was not the case in this study [17]. Also, as recommended by the GEC ESTRO work group (II), the method used to compare the two fractionation schemes was the Equivalent Dose in 2 Gy/fraction $\left(\mathrm{EQD}_{2}\right)$, because this fractionation was largely used to facilitate to interpret the results. The $\mathrm{EQD}_{2}$ formula for a treatment of ERT is [17-19]:

$$
E Q D_{2}=D \times \frac{\alpha / \beta+d}{\alpha / \beta+2}
$$

Where $d$ is the dose per fraction and $D$ represents the total dose that is equal to the dose per fraction times and the number of fractions. 
However, it is important to refer that this method is only applicable to treatments that have an interval between fractions that allows for the complete repair (6-8 hours) [17].

If this time of interruption is not present (PDR) or if a continuous irradiation occurs (LDR), it is necessary to introduce correction factors due to the incomplete repair. The formula of the $\mathrm{EQD}_{2}$ is then changed to include those factors $[2,17,20]$ :

$$
E Q D_{2}=D \times \frac{\alpha / \beta+d \times g}{\alpha / \beta+2}
$$

In the LDR case, the repair is occurring simultaneously with irradiation, the $g$ factor is [17]:

$$
g(L D R)=2 \frac{\mu \times t-1+e^{-u \times t}}{(\mu \times t)^{2}} ; \mu=\frac{\ln \times 2}{T_{1 / 2}}
$$

For short exposures, when the time $(t)$ tends towards zero, $g$ becomes 1 being equal to the formula assuming that complete repair occurs. If $t$ becomes greater (tends to infinite), $g$ tends to zero. In LDR only one fraction is generally used; in that case $d$ is equal to $D$.

With PDR, repair occurs after or at the end of the pulse, but the time between irradiations is not sufficient enough to achieve complete repair. In that case the $g$ factor is as follows [17]:

$$
\begin{gathered}
g(P D R)=\frac{2}{\mu t}\left[1-\frac{N Y-S Y^{2}}{N \mu t}\right] \\
Y=1-e^{-\mu t} \\
K=e^{-\mu x} \\
S=\frac{N K-K-N K^{2} e^{-\mu t}+K^{N+1} e^{-\mu N t}}{\left(1-K e^{-\mu t}\right)^{2}}
\end{gathered}
$$

Where $t$ is the pulse time, $x$ is the period of non-irradiation and $N$ is the number of pulses.

\section{Results}

One of the objectives of this work was the study of the influence of some factors in the biological equivalence between LDR and PDR. Using two LDR treatment schemes $(0.4$ and $0.8 \mathrm{~Gy} / \mathrm{h})$, the influence of the pulse dose and the pulse time to the dose in the calculus of the physical dose with PDR that would be equivalent to this LDR scheme was studied. For this evaluation the previous $\mathrm{EQD}_{2}$ formulas were used. For the comparison between the two techniques, " $\mathrm{L}$ " was added for the parameter corresponding to LDR and "P" for PDR.

Except for the total $\mathrm{EQD}_{2}$ of the preformed treatments, where the actual OAR doses were evaluated, it was assumed for all the calculations that the dose in the OAR was 70\% of the prescribed dose.

\section{Phase 1: Influence of physical parameters in the biological equivalent dose}

To calculate the dose with PDR equivalent to the LDR schemes referred above, the $\mathrm{EQD}_{2}$ for PDR must be equal to the LDR. Using the previous formulas we've obtained that:

$$
D_{p}=D_{L} \frac{d_{L} \times g_{L}+\alpha / \beta}{d_{p} \times g_{p}+\alpha / \beta}
$$

Where $D_{L}$ is $20 \mathrm{~Gy}, d_{L}$ is 0.4 or $0.8 \mathrm{~Gy} / \mathrm{h}$; the incomplete factors $g_{L}$ and $g_{p}$ depend on the total time (50 or $25 \mathrm{~h}$ according to the dose rate), the pulse time is equal to $0.3 \mathrm{~h}$ and the time of interruption is $0.7 \mathrm{~h}$. The $\alpha / \beta$ is $10 \mathrm{~Gy}$ for tumors and $3 \mathrm{~Gy}$ for late responding tissues, the $\mathrm{T}_{1 / 2}$ assumed is 1.5 h. Using the Microsoft ${ }^{\circledR}$ Office Excel ${ }^{\circledR} 2007$ version 12.0.6535.5002, we've calculated the dose with PDR equivalent to the referred LDR schedules.

\section{Influence of the pulse dose to equivalent dose}

We studied the influence of the pulse dose with PDR equivalent to the dose with LDR (for 0.4 and $0.8 \mathrm{~Gy} / \mathrm{h}$ ). Analyzing the results shown (Fig. 1) it was possible to conclude that only if the pulse dose was lower to the point where the two curves coincide, i.e. the total dose with PDR was the same for the tumor and for late responding tissues, it became possible to achieve the same tumor control with a reduction of the late effects or a higher tumor control with the same complications rate.

The dose rate for which the same total dose equivalent was achieved for $\alpha / \beta$ equals to 3 and $10 \mathrm{~Gy}$ was obtained by equating the $d_{P}$ formula previously analyzed for those $\alpha / \beta$ values:

$$
d_{p}=\frac{3 a-10}{g_{p}(1-a)} ; a=\frac{d L \times g_{L}+10}{d_{L} \times g_{L}+3}
$$

Assuming all the parameters referred before, the maximum pulse dose at which there would be no worsening of therapeutic control neither higher probability of complications was 0.397 and $0.792 \mathrm{~Gy} /$ pulse to be equivalent to 0.4 and 0.8 Gy/h LDR scheme, respectively, which means an decrease in the pulse dose of about $1 \%$ comparing to the dose rate with LDR. If $0.8 \mathrm{~Gy} / \mathrm{h} \mathrm{LDR}$ was used and it was intended to change to $0.7 \mathrm{~Gy} /$ pulse PDR, the equivalent physical dose with PDR corresponding to 20 Gy with LDR was $21 \mathrm{~Gy}$ for tumor and for late responding tissues, which means a necessary increase in the total dose, so that no loss in tumor control neither an increase in late response tissues effects is expected.

\section{Influence of the pulse time}

The influence of the pulse time in the dose equivalent was studied in order to determine if a correction should be applied in terms of total dose as the activity of the source diminishes and the pulse time extends, if so, what should be the value of this correction.

It is possible to visualize in Fig. 2 that the dose equivalent slightly increases as the pulse time gets larger, and the increase is more significant in the late tissues than in the tumor, however, the difference between the extremes was not higher than $1 \%$. 


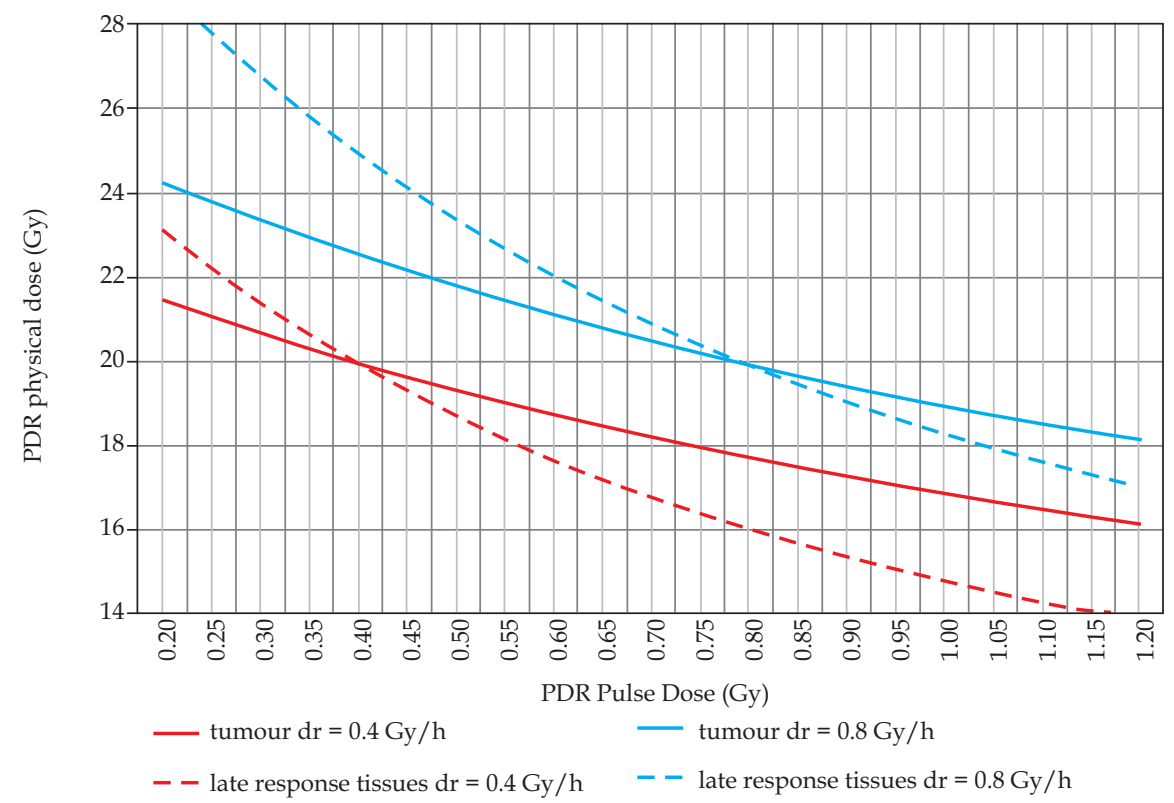

Fig. 1. Physical dose with PDR equivalent to $20 \mathrm{~Gy}$ in with 0.4 and $0.8 \mathrm{~Gy} / \mathrm{h}$ LDR for tumor $(\alpha / \beta=10)$ and late response tissues $(\alpha / \beta=3)$ varying the pulse dose between 0.2 and $1.2 \mathrm{~Gy} . \mathrm{dr}=\mathrm{LDR}$ dose rate

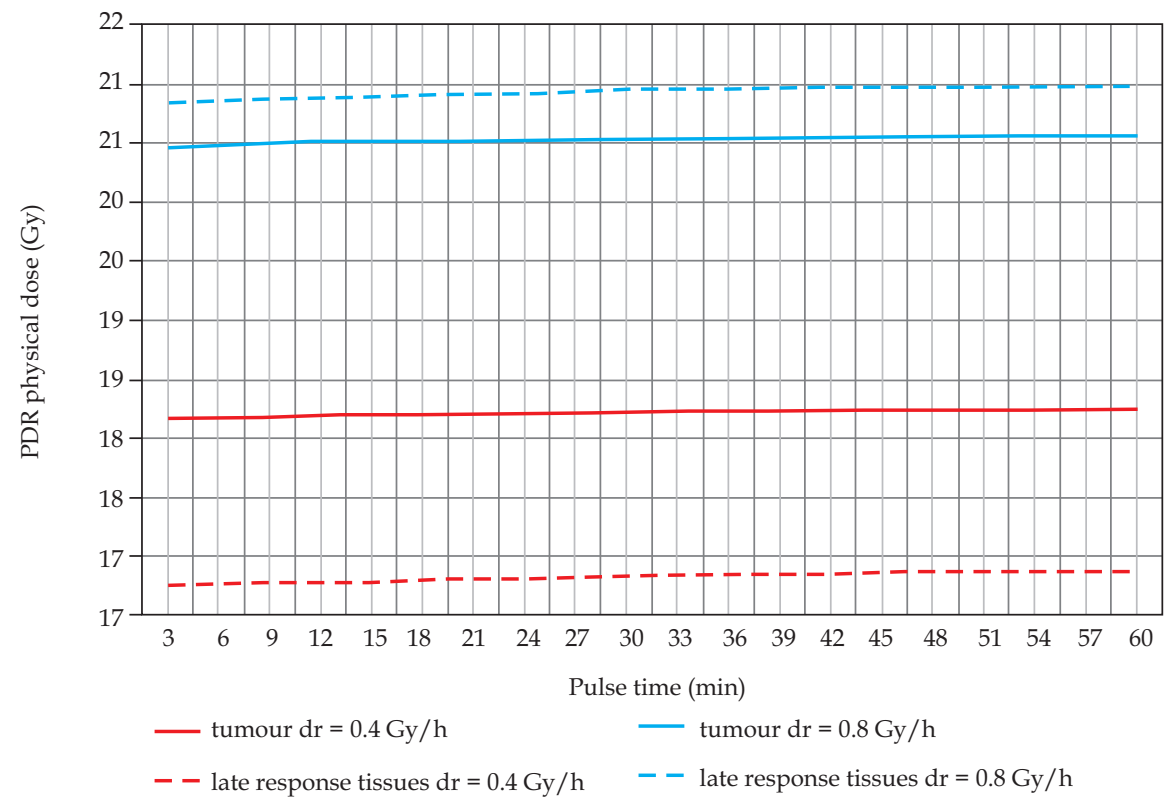

Fig. 2. Physical dose with 0.7 Gy/pulse PDR equivalent to 20 Gy with 0.4 and 0.8 Gy $/$ h LDR schemes for tumor $(\alpha / \beta=10)$ and late response tissues $(\alpha / \beta=3)$ varying the time of pulses between 3 and 60 minutes. $d r=$ LDR dose rate

\section{$\alpha / \beta$ ratios and repair half-time sensitivity analysis}

Tissues with lower $\alpha / \beta$ ratios were more sensitive to changes in the dose rate, this event is presented in Fig. 3, where the relation between the equivalent dose with PDR for four different $\alpha / \beta$ ratios was planed (3, 5, 7 and 10 Gy) [21].

The same procedure was executed for some repair half-time values $(1,1.5,2$ and $2.5 \mathrm{~h})$ [21] and we concluded that this parameter also changes the sensitivity of the tis- sue to modify the dose rate: low repair half-time is associated with low sensitivity of modifications in the dose rate; this relation is shown in Fig. 4.

Phase 2: Comparison of the total $E Q D_{2}$ for $L D R$ and PDR treatments

The dose distribution depended on several factors such as the type of applicator, the length and angles used, patient 


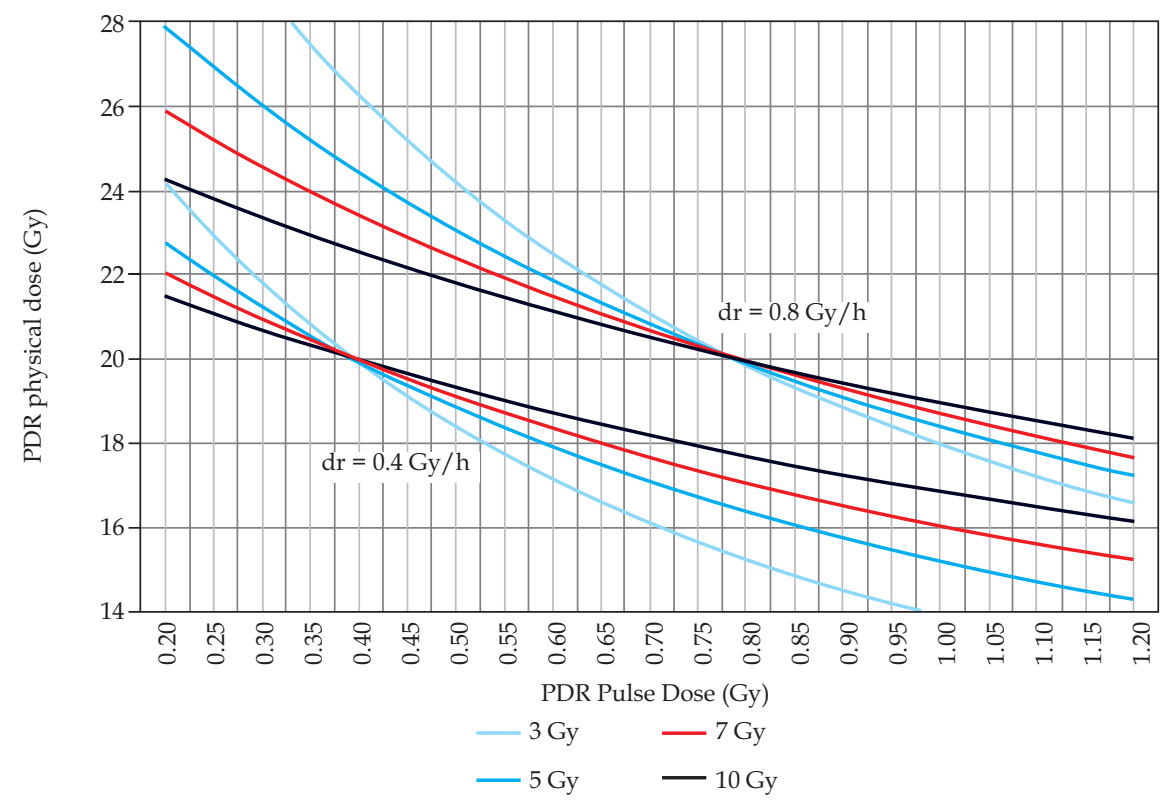

Fig. 3. Physical dose with PDR equivalent to $20 \mathrm{~Gy}$ in with 0.4 and $0.8 \mathrm{~Gy} / \mathrm{h}$ LDR for several $\alpha / \beta$ values (3, 5, 7 and 10 Gy) varying the pulse dose between 0.2 and $1.2 \mathrm{~Gy} . \mathrm{dr}=\mathrm{LDR}$ dose rate

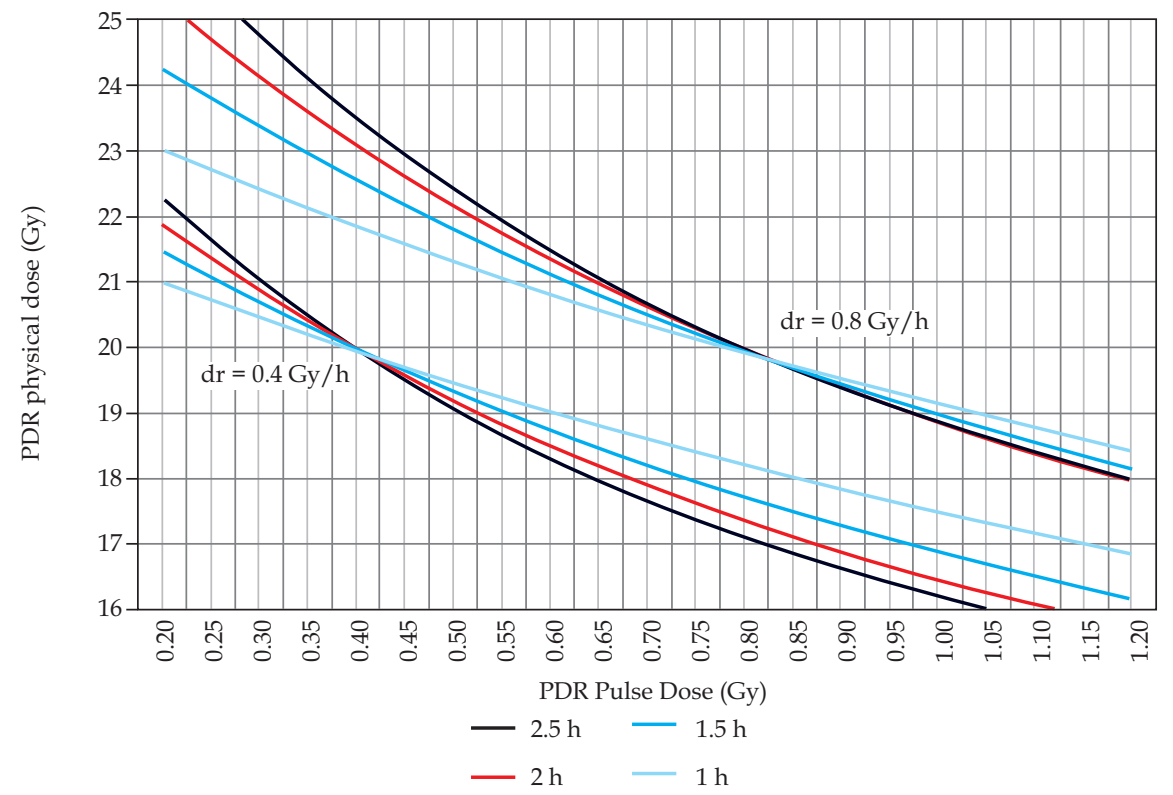

Fig. 4. Physical dose with PDR equivalent to $20 \mathrm{~Gy}$ in with 0.4 and $0.8 \mathrm{~Gy} / \mathrm{h}$ LDR for several $\mathrm{T}_{1 / 2}$ values $(1,1.5,2$ and $2.5 \mathrm{~h})$ varying the pulse dose between 0.2 and $1.2 \mathrm{~Gy} . \mathrm{dr}=\mathrm{LDR}$ dose rate

anatomy, applicator positioning, type of source, radioisotope and more [7]. For example, since a stepping source was used with PDR and a train of sources was applied with LDR, it was possible to optimize the dose distribution in PDR what was expected to be an advantage for this technique. Nowadays, several new methods of optimization are available such as MRI assisted planning and inverse planning systems that allows to achieve better dose distribution, which provides better results (tumor control and secondary effects) [3, 4].
The assumed LDR treatment schemes were used to study how the equivalent dose changes with some variations introduced, although, the unique way to evaluate treatments was to collect the treatments data from both techniques and convert total doses in comparable doses. According to the GEC-ESTRO recommendations, for comparison of treatments the total dose should be converted into $\mathrm{EQD}_{2}$; another advantage of the conversion to equivalent dose was that BT dose can be summed with the EBRT dose to calculate the 
total EQD 2 . All the analyzed patients had stage IIB cervix cancer and underwent EBRT and two BT fractions (either LDR or PDR). The patients were grouped according to BT technique: 26 were treated with LDR and 22 with PDR. It is important to refer that since we started using PDR at our Institution, all patients who underwent this treatment were included in this study.

\section{Comparison of LDR and PDR total EQD}

The same version of Microsoft ${ }^{\circledR}$ Office Excel ${ }^{\circledR}$ was used to the LQM calculations and for statistical analysis the selected software was the IBM ${ }^{\circledR}$ SPSS ${ }^{\circledR}$ Statistics v19. The total EQD 2 for each structure (tumor, rectum and bladder) and for each patient the average total $\mathrm{EQD}_{2}$ for the tumor was calculated as 89 Gy for the LDR group and 86 Gy for the PDR group, for rectum 73 Gy versus $68 \mathrm{~Gy}$ and for bladder 80 Gy versus 73 Gy $(p<0.05)$. The student $t$ test for independent samples was used to compare both groups and this conclusion was confirmed with a non-parametric test (Mann-Whitney U).

To exclude the hypothesis that the difference observed could be due to the EBRT, the EQD ${ }_{2}$ for this technique was statistically analyzed. The same statistical test was applied and it was concluded that the total $\mathrm{EQD}_{2}$ differences between LDR and PDR were not due to EBRT. The EBRT EQD ${ }_{2}$ in the PDR treatment were higher than in the LDR treatment group, however the differences were not bigger than 1.9 Gy and they were not statistically significant $(p>0.05)$. The total $\mathrm{EQD}_{2}$ difference between the groups relies only on BT technique. The means and confidence intervals for the $\mathrm{EQD}_{2}$ for the total treatment and the EBRT phase are presented in Fig. 5.

\section{Correction to the PDR prescription dose}

The calculations made previously to disclosure the dose with PDR equivalent to a certain LDR scheme were repeated using these average values from the collected data. The average prescribed dose for the patients treated with LDR was $21 \mathrm{~Gy}$ and the treatment time was $23 \mathrm{~h}$. For the PDR technique the average pulse time of $0.37 \mathrm{~h}$ was used. It was calculated that the dose with PDR equivalent to the $21 \mathrm{~Gy}$ in $23 \mathrm{~h}$ with a pulse time of $0.37 \mathrm{~h}$ was $22 \mathrm{~Gy}$ for tumor $(\alpha / \beta=10 \mathrm{~Gy})$ and $23 \mathrm{~Gy}$ for late responding tissues $(\alpha / \beta=3$ Gy). In summary, the dose with PDR ought to be between 6 to $10 \%$ higher to be equivalent to the treatment with LDR at $0.9 \mathrm{~Gy} / \mathrm{h}$.

\section{Discussion}

If two schemes of treatment are being compared and they are said to be equivalent, that means that they are equivalent for certain effect, although, not probable to achieve treatment equivalence for all the effects [2]. If the PDR dose rate used was the same as applied in the LDR, that would imply a slightly higher late effects or a small loss of tumor control, because the equivalent dose for late responding tissues is lower than for tumor. For the LDR schemes analyzed in the phase 1 and a pulse time assumed to be $0.3 \mathrm{~h}$ it was possible to achieve the PDR treatment that was equivalent both for tumor and late response effects. To do so, it was necessary to reduce the dose rate in $1 \%$ to maintain the same total dose. If the dose rate was lowered bellow that $1 \%$, a therapeutic benefit is expected, since late responding tissues were more sensitive to a change in dose rate than acute responding tis-

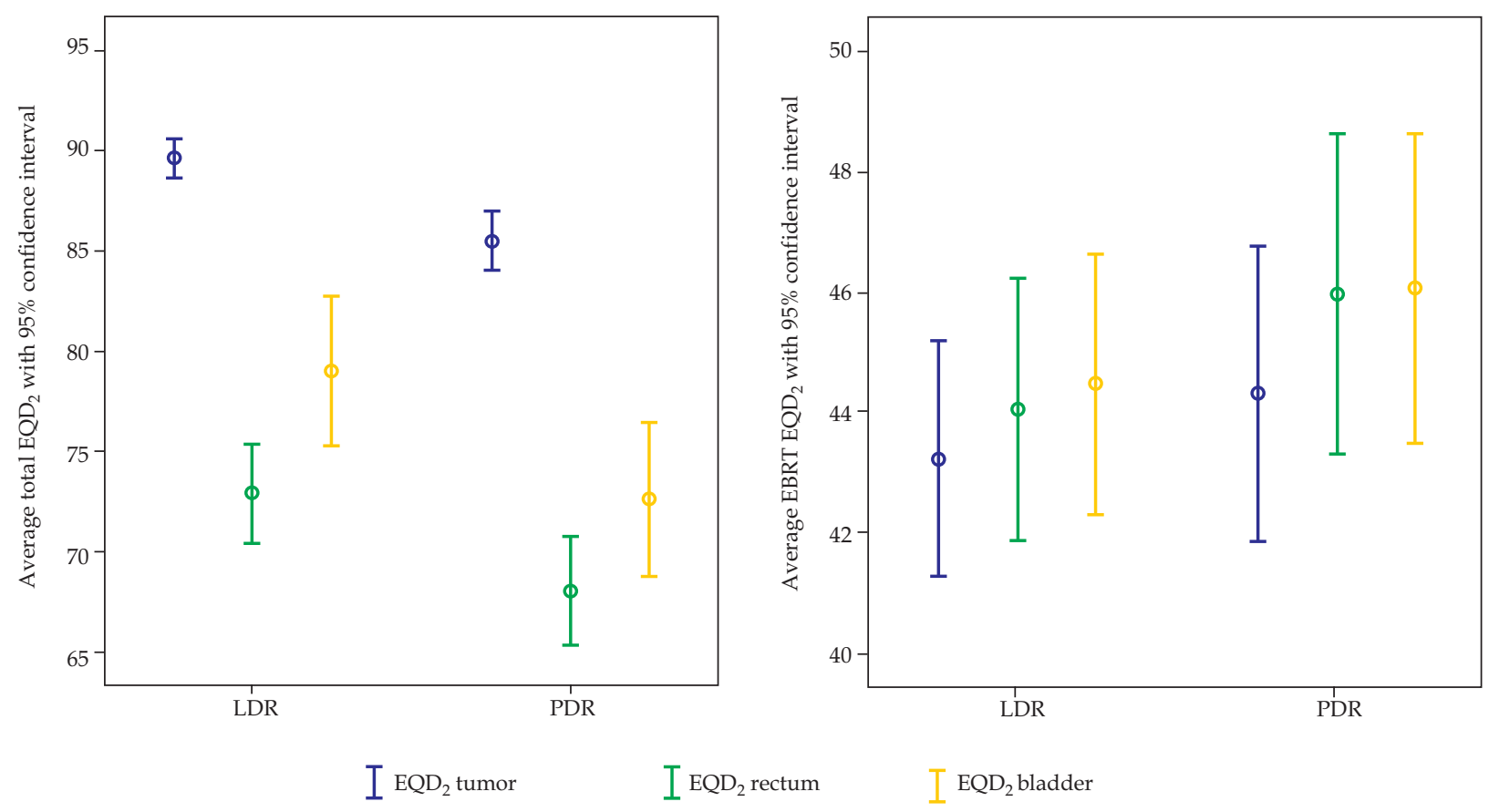

Fig. 5. In the left graphic the total $\mathrm{EQD}_{2}$ for each structure and technique is shown. With PDR, the total $\mathrm{EQD}_{2}$ is lower than $\mathrm{LDR}$ for all structures. In the right are the $\mathrm{EQD}_{2}$ values for the EBRT phase; is not possible to observe a significant difference between the two groups 
sues. However, this reduction in dose rate ought to be accompanied by a correction in the total prescribed dose. In treatments with higher pulse doses it was necessary to reduce the total dose as it has been previously shown [14]. In our work we demonstrated that with lower pulse doses, higher total doses are needed.

The influence of the pulse time in the equivalent dose was studied and we concluded that the correction needed between a treatment with a pulse time of $0.05 \mathrm{~h}$ and another one using $1 \mathrm{~h}$ was less than $1 \%$, therefore, no correction for pulse time was necessary. The use of an intermediate value was suggested or the average pulse time of the PDR treatments could also be used. When comparing total $\mathrm{EQD}_{2}$ between the LDR and PDR groups, we observed that the techniques were not equivalent. The average total $\mathrm{EQD}_{2}$ was lower with PDR than LDR for tumor, rectum and bladder. We also concluded that the difference was not due to the EBRT. On one hand a greater protection of late responding tissues was expected, on the other hand, a loss of tumor control was likely to occur.

\section{Conclusions}

In summary, we concluded that if 0.7 Gy/pulse PDR treatment was used to substitute $0.9 \mathrm{~Gy} / \mathrm{h}$ LDR treatment, an increase in the total dose (6 to $10 \%$ increase) was necessary. It is important to mention that this linear correction was only valid for the average LDR treatment, $21 \mathrm{~Gy}$ in $23 \mathrm{~h}(0.9 \mathrm{~Gy} / \mathrm{h}) \mathrm{LDR}$ treatment, replaced by a PDR scheme with $0.7 \mathrm{~Gy} /$ pulse. The linear correction could be used as a reference, because those parameters were the average treatment parameters, however, it is recommended that the equivalence for the desired LDR scheme should be calculated using the LQM. This procedure could be executed using a spreadsheet once the process is fast and efficient. A spreadsheet could also be used to calculate the total $\mathrm{EQD}_{2}$ for registration as recommended by GEC ESTRO [17, 22]. Although these two techniques were not equivalent, their prescribed total $\mathrm{EQD}_{2}$ values were comparable (89 Gy vs. $86 \mathrm{~Gy})$ and were within the range presented in other studies [23] and the total $\mathrm{EQD}_{2}$ for the rectum and bladder were within the ABS recommendations (80 Gy for bladder and 75 Gy for rectum) [11, 24].

\section{Acknowledgements}

We would like to thank Dr. Lurdes Trigo, director of the Brachytherapy Service of the Oporto Oncology Institute, for the support given to this study. No funds were received for the execution of this work.

The research was conducted in the Department of Brachytherapy at Instituto Português de Oncologia Francisco Gentil Porto E.P.E. Address: Rua Dr. António Bernardino de Almeida 4200-072 Porto, Portugal.

\section{References}

1. Gerbaulet A, Ash D, Meertens H. General Aspects. In: Gerbaulet A, Potter R, Mazeron J et al. The GEC ESTRO handbook of Brachytherapy. ESTRO Editions, Leuven 2002; 3-21.

2. Mayles P, Nahum A, Rosenwald JC et al. Handbook of radiotherapy physics - Theory and practice. Taylor and Francis, Paris 2007; 1093-1204.
3. Petric P, Hudej R, Music M. MRI assisted cervix cancer brachytherapy pre-planning, based on insertion of the applicator in para-cervical anesthesia: preliminary results of a prospective study. J Contemp Brachyther 2009; 1: 163-169.

4. Trnková P, Baltas D, Karabis A et al. A detailed dosimetric comparison between manual and inverse plans in HDR intracavitary/intersticial cervical cancer brachytherapy. J Contemp Brachyther 2010; 2: 163-170.

5. Sharma B, Singh T, Singh T. Evaluation of variation in dose of organs at risk in intracavitary brachytherapy of cervical cancer - a prospective study. J Contemp Brachyther 2011; 3: 23-25.

6. Gerbaulet A, Potter R, Haie-Mader C. Cervix Carcinoma. In: Gerbaulet A, Potter R, Mazeron J et al. The GEC ESTRO handbook of Brachytherapy. ESTRO Editions, Leuven 2002; 301363.

7. Mazeron J, Scalliet P, Limbergen E et al. Radiobiology of Brachytherapy and the Dose-Rate Effect. In: Gerbaulet A, Potter R, Mazeron J et al. The GEC ESTRO handbook of Brachytherapy. ESTRO Editions, Leuven 2002; 95-109.

8. ICRU Report 38 - Dose and Volume Specifications for Reporting Intracavitary Therapy in Gynecology. International Commission on Radiation Units and Measurements. 1985

9. Meertens H, Briot E. Radiophysics. In: Gerbaulet A, Potter R, Mazeron J et al. The GEC ESTRO handbook of Brachytherapy. ESTRO Editions, Leuven 2002; 23-57.

10. Hunter RD, Davidson SE. Low dose-rate brachytherapy for treating cervix cancer: changing dose rate. In: Joslin CAF, Flynn A, Hall E. Principles and practice of brachytherapy using afterloading systems. Arnold, London 2001; 343-353.

11. Nag S, Chao C, Erickson B et al. The American Brachytherapy Society recommendations for low-dose-rate brachytherapy for carcinoma of the cervix. Int J Radiat Oncol Biol Phys 2002; 52: 33-48.

12. Brenner D, Hall E, Randers-Pehrson G et al. Quantitative comparisons of continuous and pulsed low dose rate regimens in a model late-effect system. Int J Radiat Oncol Biol Phys 1996; 34: 905-910.

13. Visser A, Van Den Aardweg G, Levendag A. Pulsed dose rate and fractionated high dose rate brachytherapy: choice of brachytherapy schedules to replace low dose rate treatments. Int J Radiat Oncol Biol Phys 1996; 34: 497-505.

14. Hall E, Brenner D. Pulsed dose rate brachytherapy: can we take advantage of new technology? Int J Radiat Oncol Biol Phys 1996; 34: 511-512.

15. Fowler J, Van Limbergen E. Biological effect of pulsed dose rate brachytherapy with stepping sources if short half-times of repair are present in tissues. Int J Radiat Oncol Biol Phys 1997; 37: 877-83.

16. Bachtiary B, Dewitt A, Pintilie M et al. Comparison of late toxicity between continuous low dose rate and pulsed dose rate brachytherapy in cervical cancer patients. Int J Radiat Oncol Biol Phys 2005; 63: 1077-1082.

17. Potter R, Haie-Meder C, Van Limbergen E. Recommendations from gynaecological (GYN) GEC ESTRO working group (II): Concepts and terms in 3D image-based treatment planning in cervix cancer brachytherapy - 3D dose volume parameters and aspects of 3D image-based anatomy, radiation physics. Radioth Oncol 2006; 78: 1-16.

18. Thames H, Withers H, Peters L et al. Changes in early and late radiation responses with altered dose fractionation: implication for dose-survival relationships. Int J Radiat Oncol Biol Phys 1982; 8: 219-226.

19. Withers H, Thames H, Peters L. A new isoeffect curve for change in dose per fraction. Radiother Oncol 1983; 1: 187-191.

20. Van der Kogel A, Joiner M. Basic Clinical Radiobiology. Edward Arnold Editors, London 2009; 102-134. 
21. Joiner M, Bentzen S. Fractionation: the linear-quadratic approach. In: Joiner M, Van der Koegel A. Basic Clinical Radiobiology. Arnold 2009; 102-119.

22. Lang S, Kirisits C, Dimopuolos J et al. Treatment planning for MRI assisted brachytherapy of gynecologic malignancies based on total dose constraints. Int J Radiat Oncol Biol Phys 2007; 69: 619-627.

23. Lang $S$, Nulens A, Briot $E$ et al. Intercomparison of treatment concepts for MR image assisted brachytherapy of cervical carcinoma based on GYN GEC-ESTRO recommendations. Radiother Oncol 2006; 78: 185-193.

24. Nag S, Erickson B, Thomadsen B et al. The American Brachytherapy Society recommendations for high-dose-rate brachytherapy for carcinoma of the cervix. Int J Radiat Oncol Biol Phys 2000; 48: 201-211. 